\title{
CIÊNCIA RESPONSÁVEL DOS DADOS: IMPARCIALIDADE, PRECISÃO, CONFIDENCIALIDADE E TRANSPARÊNCIA DOS DADOS
}

\author{
RESPONSIBLE DATA SCIENCE: IMPARTIALITY, \\ ACCURACY, CONFIDENTIALITY AND TRANSPARENCY \\ OF DATA
}

\author{
Morgana Carneiro Andradea \\ Paula Regina Ventura Amorim Gonçalez ${ }^{b}$ \\ Decio Wey Berti Junior \\ Ana Alice Baptistad \\ Caio Saraiva Conegliane
}

\begin{abstract}
RESUMO
Introdução: no contexto Big Data, surge, como necessidade urgente, a aplicação de direitos individuais e empresariais e de normas regulatórias que resguardem a privacidade, a imparcialidade, a precisão e a transparência. Nesse cenário, a Responsible Data Science desponta como uma iniciativa que tem como base as diretrizes FACT, que correspondem à adoção de quatro princípios: imparcialidade, precisão, confidencialidade e transparência. Objetivo: abordar alternativas que podem assegurar a aplicação das diretrizes FACT. Metodologia: foi desenvolvida investigação exploratória e descritiva com abordagem qualitativa. Foram realizadas pesquisas nas bases de dados bibliográficas Web of Science, Scopus e pelo motor de busca Scholar
\end{abstract}

a Doutorado em Tecnologias e Sistemas de Informação pela Universidade do Minho (UMINHO). Bibliotecária na Universidade Federal do Espírito Santo (UFES). E-mail: morganaandrade@hotmail.com

b Doutora em Ciência da Informação pela Universidade Estadual Paulis Júlio de Mesquita Filho (UNESP). Professora do Curso de Biblioteconomia da Universidade Federal do Espírito Santo (UFES). E-mail: paulaventuraamorim@gmail.com

c Doutor em Gestão e Organização do Conhecimento pela Universidade Federal de Minas Gerais (UFMG). Analista de Sistemas na Assessoria de Tecnologia de Informação da Universidade Estadual de Londrina (UEL). E-mail: deciowbj@gmail.com

${ }^{d}$ Doutora em Engenharia de Sistemas de Informação pela Universidade do Minho (UMINHO). Professora do Departamento de Sistemas de Informação, Universidade do Minho. E-mail: analice@dsi.uminho.pt

e Doutor em Ciência da Informação pela Universidade Estadual Paulis Júlio de Mesquita Filho (UNESP). E-mail: caio.coneglian@gmail.com 
Google com a utilização dos termos "Responsible Data Science", "Fairness, Accuracy, Confidentiality, Transparency + Data Science", FACT e FAT relacionados com Data Science. Resultados: a Responsible Data Science desponta como uma iniciativa que tem como base as diretrizes FACT, que correspondem à adoção dos princípios: imparcialidade, precisão, confidencialidade e transparência. Para a implementação dessas diretrizes, deve-se considerar o uso de técnicas e abordagens que estão sendo desenvolvidas pela Green Data Science. Conclusões: concluiu-se que a Green Data Science e as diretrizes FACT contribuem significativamente para a salvaguarda dos direitos individuais, não sendo necessário recorrer a medidas que impeçam o acesso e a reutilização de dados. Os desafios para implementar as diretrizes FACT requerem estudos, condição sine qua non para que as ferramentas para análise e disseminação dos dados sejam desenvolvidas ainda na fase de concepção de metodologias.

Descritores: Ciência dos dados. Ética. Big data. Ciência Responsável dos Dados.

\section{INTRODUÇÃO}

O aumento do volume de dados tem modificado a maneira como pesquisas, governança, socialização e negócios estão sendo realizados (HILBERT; LÓPEZ, 2011; KEMPER; KOLKMAN, 2018). Podem ser gerados por diferentes segmentos da sociedade, governo, indústria, institutos de pesquisa e universidades. São coletados, armazenados e divulgados de diferentes maneiras e por diferentes organismos (EUROPEAN DATA SCIENCE ACADEMY, 2019; STOYANOVICH; HOWE, 2018; TAYLOR, 2017).

Exemplos recentes de coletas e utilização de dados têm sido amplamente noticiados: o uso de dados referentes ao registro digital e bibliométrico pela Índia e pela China com o intuito de rastrear e monitorar os cidadãos (TAYLOR, 2017); a coleta de dados pessoais do Facebook, utilizados para fins políticos, sem consentimento dos usuários, pela Cambridge Analytica (FACEBOOK, 2019); a utilização dos dados dos usuários do Google Maps e Youtube pelo Google para enviar anúncios personalizados (SATARIANO, 2019); e o compartilhamento de dados por meio de Application Programming Interface (API) (PIERSMA, 2018). Ações como essas estão relacionadas com a preocupação quanto ao uso e à publicação de dados no que concerne ao acesso e à divulgação de informações privadas ou públicas e à privacidade ou conclusões injustas e/ou tendenciosas por parte de diferentes atores (AIMS, 2017; OHM, 2014; TAYLOR, 2017). São fatos associados às novas "Webs", à "Internet das Coisas (IoT)", à "Web de 


\section{Dados", ao Big Data.}

A demanda por acesso e análise de dados privados e públicos tem aumentado de forma a chamar a atenção de vários segmentos da sociedade. $\mathrm{O}$ panorama atual mostra que empresas obtêm lucro, governos influenciam a tomada de decisão em relação aos seus cidadãos e pessoas são induzidas a conceitos cujo agravante é que nem sempre os dados que subsidiam essas ações são imbuídos dos princípios da ética e da justiça (KEMPER; KOLKMAN, 2018; STOYANOVICH; HOWE, 2018; TAYLOR, 2017)

Em meio a essa problemática, surge, como forte demanda, a aplicação de direitos individuais e empresariais e de normas regulatórias que resguardam a privacidade do indivíduo e a transparência das informações (MOEREL; PRINS, 2016). Teve início, nos anos de 2016-2017, um movimento em busca da imparcialidade, responsabilidade e transparência (FAT) na tomada de decisão algorítmica e na ciência dos dados de forma mais ampla (STOYANOVICH; HOWE; JAGADISH, 2018). Nesse sentido, surge o projeto Responsible Data Science (RDS), Ciência Responsável de Dados, apresentada como possibilidade de equacionar questões em relação à imparcialidade, precisão, confiabilidade e transparência, no que se refere ao Big Data e à Ciência dos Dados. Esses quatro princípios são representados pelo acrônimo FACT (Fairness, Accuracy, Confidentiality, Transparency) (VAN DER AALST, 2016).

O RDS busca desenvolver métodos e técnicas para apoiar a publicação e o uso de dados de acordo com o FACT, com o objetivo de propiciar tecnologia para garantir esses princípios ainda na etapa de desenvolvimento e criar plataformas multidisciplinares. A adoção do FACT, ainda na fase de concepção das tecnologias e métodos aplicados ao Big Data e à Ciência dos Dados, apresenta-se como alternativa para garantir a qualidade e segurança na utilização de dados (STOYANOVICH; HOWE, 2018; VAN DER AALST, 2016).

Diante dos desafios de incorporar valores éticos de forma justa e sustentável aos direitos individuais conforme as inovações tecnológicas que envolvem a Ciência dos Dados, o presente estudo busca identificar orientações e iniciativas que viabilizem a implementação das diretrizes FACT por aqueles 
que trabalham nesse contexto.

Este artigo está estruturado em quatro seções: Introdução; Seção 1, onde é apresentado o tema; Seção 2, que contém a abordagem teórica em torno da Responsible Data Science e das diretrizes FACT; os procedimentos metodológicos são descritos na Seção 3; os resultados obtidos são elencados na Seção 4; e, ao final, são apresentados as Considerações Finais, Referências e Glossário.

\section{REFERENCIAL TEÓRICO}

Para Mayo (1996) e Chalmers (2013), citados por van der Aalst (2016), no contexto da Web há uma mudança na forma como o conhecimento é gerado. Quando o conhecimento é baseado em dados, "[...] segue a lógica do novo experimentalismo, em que o conhecimento é derivado de observações experimentais, não da teoria". Nesse novo paradigma, surgem preocupações em relação aos dados, principalmente como eles podem ser utilizados de forma irresponsável.

Um grupo de pesquisadores de diferentes instituições na Holanda deu início a um novo preceito: RDS que, segundo van der Aalst (2016), visa a produzir soluções positivas ao invés de evitar o uso dos dados. O consórcio RDS foi lançado em 2016 com o objetivo de "[...] combater desafios éticos e legais, e desenvolver técnicas de ciência de dados, infraestruturas e abordagens responsáveis por processos justos, precisos e dados confidenciais, e transparentes por concepção" (AIMS, 2017; MOEREL; PRINS, 2016).

Essa iniciativa envolve disciplinas, como processo de mineração de dados, humanidades digitais, ética, recuperação da informação, representação do conhecimento, direito, machine learning, processamento de linguagem natural, segurança, estatística e visualização (VAN DER AALST, 2016, VAN DER AALST; BICHLER; HEINZL, 2017) e pode ser aplicada nos domínios de mídia, segurança e saúde.

Para a Academia Europeia de Ciência dos Dados, "A 'Era dos Dados' produz uma diversidade de dados de forma exponencial cujos desafios estão 
relacionados à recolha, armazenamento e análise desses dados" (http://edsaproject.eu/overview/aboutedsa/). Nesse ambiente, têm sido desenvolvidas técnicas poderosas, como mineração de dados, aprendizado de máquina, banco de dados e visualização, que objetivam melhorar a vida de pessoas com a oferta de novos serviços e produtos com maior qualidade e eficiência (GE 301 GROUP 7, 2017; STOYANOVICH et al., 2017; STOYANOVICH; HOWE; JAGADISH, 2018). Na visão de Piersman (2018), a RDS “[...] é um dos fundamentos da transformação digital".

No entanto, decisões automáticas de dados podem ser injustas e não transparentes; dados confidenciais podem ser compartilhados involuntariamente ou utilizados de forma indevida por terceiro; imprecisões podem ser geradas a partir de incorporação de vieses por parte de algoritmos, entre outros problemas (OHM, 2014; STOYANOVICH; HOWE, 2018). Esses fatos provocam grande preocupação em relação à produção e uso dos dados, nomeadamente no que concerne a informações falsas, conclusões preconceituosas, divulgação de informações privadas e não transparentes, o que pode prejudicar a aplicação de dados científicos (AIMS, 2017), por exemplo, ou ainda comprometer a segurança das pessoas e a vida pessoal e profissional do indivíduo (GE 301 GROUP 7, 2017; OHM, 2014; TAYLOR, 2017).

Exemplos sobre algumas dessas ocorrências são identificados no nosso dia a dia da forma mais simples a mais complexa e podem provocar sérias consequências tanto para os prestadores de serviços como para os proprietários dos dados (FACEBOOK, 2019; SATARIANO, 2019). Um exemplo de como facilmente se pode chegar a uma conclusão preconceituosa pode ser observado a partir de uma simples busca realizada no Google para definição do termo professores (Figura 1). 
Figura 1- Pesquisa no Google

\begin{tabular}{l|l}
\hline Gafle & professors are \\
\hline professors are prejudiced too \\
professors are overpaid \\
professors are rude \\
professors are losing their freedom of expression \\
professors are bad teachers \\
professors are jerks \\
professors are idiots \\
professors are busy \\
professors are tenured \\
professors are out of touch \\
unversıa... \\
Setor de atividade: Magistério
\end{tabular}

Fonte: Google

Richard e King (2014), referenciados por Lodder (2016), professor da Vrije Universiteit, Amsterdam, considera que a construção dessa nova sociedade digital, a qual ele denomina de Sociedade Big Data, e o equilíbrio de valores humanos, como privacidade, confidencialidade, transparência, identidade, livre arbítrio, associados às novas estruturas digitais, serão impactantes na construção dos indivíduos. Nesse sentido, alerta sobre a relevância de se preservar esses valores em benefício da inovação e da conveniência.

Esses valores são reiterados pela a iniciativa RDS que, como esboçado, tem por objetivo o desenvolvimento de um modelo sustentável para a ciência de dados com base nos valores éticos e aplicação de técnicas em conformidade com os requisitos de imparcialidade, responsabilidade, transparência e confidencialidade por concepção (Data Science Center Eindhoven, 2018).

Para o Data Science Center Eindhoven Research Program,

[...] os valores da sociedade são uma parte intrínseca do valor do Big Data. Construir valores na ciência de dados 'por design' é um campo de pesquisa interdisciplinar desafiador e fascinante, com muitas aplicações práticas. Criar valor social através da ciência de dados requer uma compreensão do contexto e uma governança eficaz dos dados! (DATA SCIENCE CENTER EINDHOVEN, 2018).

Assim, a Research Data Science emerge baseada em quatro pilares imparcialidade, precisão, confidencialidade e transparência -, com o objetivo de atender às questões que afetam a sociedade em diferentes aspectos, éticos, econômicos e/ou sociais (Figura 2). 


\section{Figura 2 - Research Data Science}
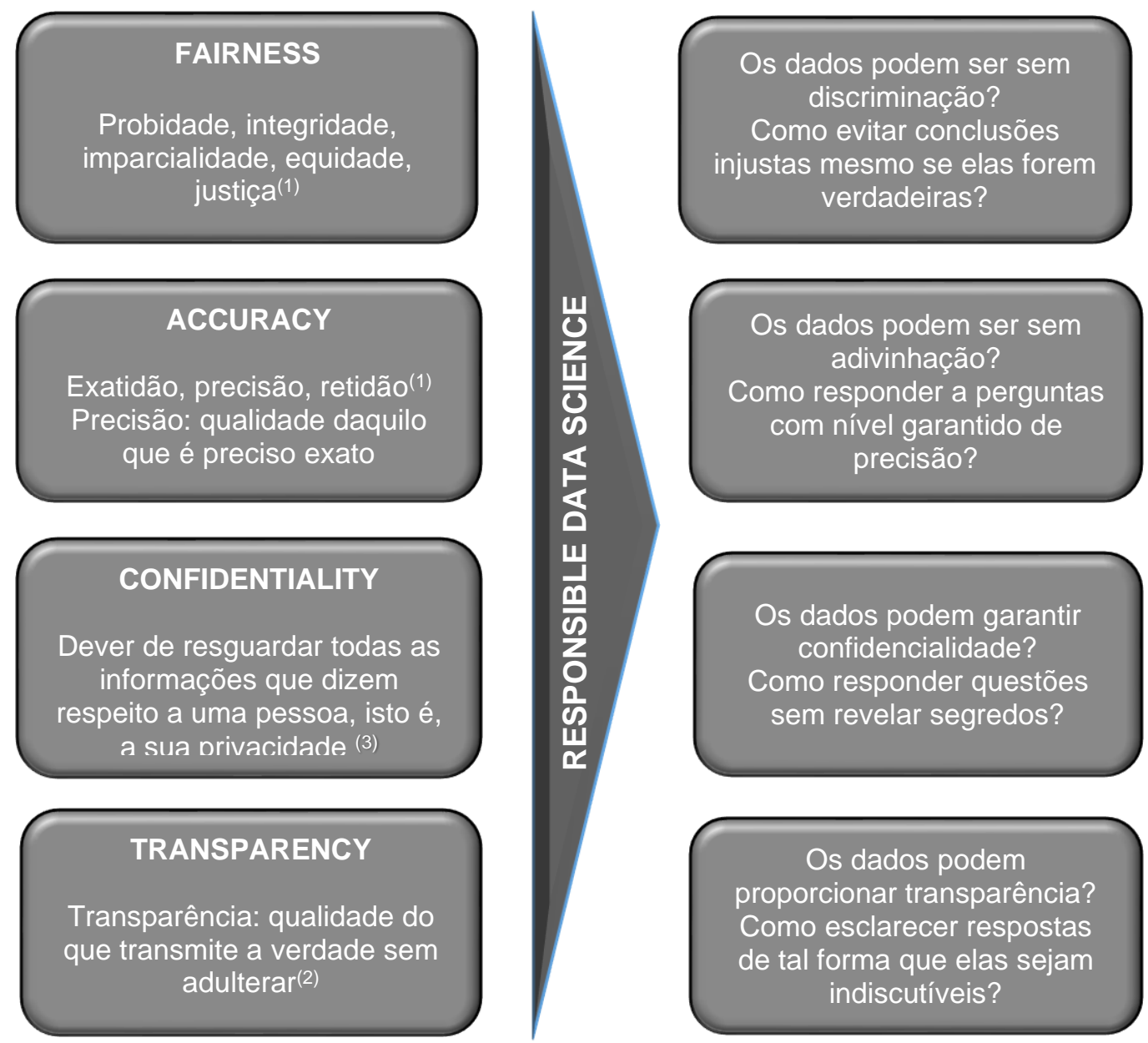

Nota: (1) Michaelis.uol.com.br; (2) Wikipédia; (3) Goldim (1997)

Fonte: Adaptado de van der Aalst (2016)

Esses princípios podem ser assim definidos:

IMPARCIALIDADE: "[...] É a atitude de quem considera, objetivamente, sem paixões ou preconceitos, um determinado fato na totalidade de seus aspectos" (ÁVILA, 1967).

A título de imparcialidade, justiça dos dados, Taylor (2017, p. 1) afirma que a disponibilidade de dados digitais, principalmente a partir do uso de dispositivos e serviços tecnológicos pelas pessoas, "[...] tem implicações políticas e práticas na maneira como as pessoas são tratadas pela comunidade, Estado e pelo setor privado". No entanto, o nível de conscientização e a implementação de mecanismos que combatam possíveis discriminações é 
menor, se comparados com a rapidez com que são desenvolvidas as tecnologias de processamento de dados.

PRECISÃO: a precisão das informações ou medições é a sua qualidade de ser verdadeira ou correta, mesmo em pequenos detalhes (COLLINS DICTIONARY, 2019).

A produção de resultados imprecisos independe da quantidade de dados analisados. Variáveis são usadas para prever um resultado. Para isso deve haver correlação positiva ou negativa entre variáveis e resultado com o objetivo de obter melhor precisão (GE 301 GROUP 7, 2017).

CONFIDENCIALIDADE: a confidencialidade é considerada como o dever de resguardar todas as informações que dizem respeito a uma pessoa, isto é, a sua privacidade. A confidencialidade inclui a preservação das informações privadas e íntimas (GOLDIM, 1997).

TRANSPARÊNCIA: a transparência pode ser entendida como a "'[...] compreensibilidade de um modelo específico'" (LEPRI et al., 2018) e é vista como um requisito para a responsabilização algorítmica. Ainda pode significar "[...] que todos os códigos e todos os dados devem estar públicos" (STOYANOVICH et al., 2018, p. 2165).

Annany e Crawford (2016)

[...] sugerem que a transparência não pode ser uma característica de um modelo algorítmico. Em vez disso, a opacidade dos algoritmos deve ser considerada com sensibilidade para os contextos de seu uso; a transparência é realizada por conjuntos sociotécnicos de algoritmos e pessoas.

No entanto, a transparência nem sempre é total, seja em razão de garantias associadas a direitos comerciais, seja em razão da privacidade dos cidadãos. O desreipeito a esses princípios é caracterizado como violação das leis (STOYANOVICH et al., 2018).

Para Kemper e Kolkman (2018),

A ciência de dados só pode ser eficaz se as pessoas confiarem nos resultados e puderem inferir e interpretar corretamente os resultados. A ciência de dados não deve, portanto, ser vista como uma caixa preta que transforma magicamente dados em valor. Muitas escolhas de projeto precisam ser feitas em um típico 'data science pipeline' [...]. 
Os princípios aqui citados são defendidos pela RDS e estão associados à legalidade, à norma e à ética que, ao juntar-se aos princípios FAIR (Findability, Accessibility, Interoperability and Reusability), surgem como os principais temas de pesquisa em Big Data. Nota-se que as diretrizes FACT vêm complementar o FAIR, principalmente por focar aspectos essenciais para a reutilização dos dados.

A discussão sobre FAIR tem envolvido profissionais e instituições de diferentes domínios e encontra-se em um estágio mais avançado, portanto com maior número de publicações. No que tange às diretrizes FACT, por terem sido apresentadas recentemente ou por ainda os atores que atuam na área não compreenderem a dimensão desses elementos, não atingiram o mesmo nível de discussão, embora suas implicações envolvam a sociedade de forma mais presente e impactante. Os defensores das diretrizes FACT enfatizam que elas devem ser aplicadas não apenas no uso de modelos algorítmicos, mas também nas fases de projeto e desenvolvimento.

Esses princípios vêm norteando ações, principalmente, dentro da Comunidade Europeia, a exemplo da recente penalidade infligida à empresa Google por "[...] falta de transparência, informação inadequada e falta de consentimento válido em relação à personalização de anúncios" (EURONEWS, 2019).

A seguir, serão apresentados os procedimentos metodológicos aplicados a este estudo, cujo objetivo é identificar contribuições e iniciativas baseadas nas diretrizes FACT.

\section{PROCEDIMENTOS METODOLÓGICOS}

Este trabalho refere-se a uma investigação descritiva em que a pesquisa bibliográfica foi realizada a partir das bases de dados Scopus e Web of Science e do motor de busca Google Scholar. A pesquisa documental foi desenvolvida em sites de organizações que abordam a presente temática: Responsible Data Science, Data Science Center Eindhoven e GE 301-Group 7. As buscas foram 
realizadas no período de 15 de dezembro de 2018 a 15 de janeiro de 2019, utilizando os termos "Responsible Data Science", "Fairness, accuracy, confidentiality, transparency + Data science", FACT e FAT relacionados com Data Science.

\section{RESULTADOS}

A pesquisa bibliográfica proporcionou a identificação de 54 documentos que abordam a iniciativa Responsible Data Science. Ao usar os termos FACT, FAT e "Fairness, accuracy, confidentiality, transparency", associados à "Data Science"/"Big Data", foram identificados 20 documentos. Consulta a sites de organizações/instituições proporcionaram a recuperação de mais 5 documentos. A partir da leitura dos documentos selecionados, encontrou-se o termo "Green Data Science", que passou a ser incluído na pesquisa bibliográfica, resultando na identificação de mais 16 , totalizando 95 documentos. Após a eliminação dos itens duplicados, análise do título e resumo para identificação da pertinência com o objetivo do estudo, foram selecionados 24 documentos para leitura (Tabela 1).

Tabela 1 - Resultado da pesquisa bibliográfica

\begin{tabular}{|l|r|r|r|r|}
\hline \multicolumn{1}{|c|}{ Termos } & Scopus & $\begin{array}{c}\text { Web of } \\
\text { Science }\end{array}$ & $\begin{array}{c}\text { Google } \\
\text { scholar }\end{array}$ & Medline \\
\hline Responsible Data Science & 7 & 1 & 40 & 6 \\
\hline FACT + Data Science & 0 & 0 & 10 & 0 \\
\hline FAT + Data Science & 1 & 2 & 0 & 0 \\
\hline $\begin{array}{l}\text { Fairness, accuracy, confidentiality, } \\
\text { transparency + Data Science }\end{array}$ & 6 & 2 & 4 & 1 \\
\hline Green data Science & 1 & 0 & 15 & 0 \\
\hline
\end{tabular}

Fonte: Autores.

Esclarecemos que a busca do termo "Responsible Data Science" no Google resultou na recuperação de 14.600 itens, que incluem blogs, lectures, apresentações em slide, entrevistas, posts no Twitter, artigos (recuperados via Google Scholar). Em vista da grande quantidade, com pouca precisão, e pela diversidade de tipo de publicações, não foram incluídos os resultados obtidos com a busca no Google, apenas os identificados no Google Scholar.

A partir da análise dos 24 documentos, foram extraídas informações 
quanto ao tipo de publicação, de pesquisa, conceitualização dos termos "Responsible Data Science", "Green Data Science", "Fairness", "Accuracy", "Confidentiality", "Transparency" e identificação de iniciativas/projetos alinhados à RDS.

Observamos que, embora os termos que representam o acrônimo FACT possam trazer conceitos ambíguos, a depender do contexto e do domínio, eles foram pouco discutidos em relação à semântica pelos autores dos artigos. Nesse sentido, ao final deste estudo, é apresentado um glossário com esses termos e com os termos transversais.

A iniciativa Responsible Data Science representa um grupo que procura alternativas para a implantação do FACT. As ações em busca de resultados são contempladas pela Green Data Science, termo cunhado por um dos fundadores do grupo RDS.

Dos 24 documentos analisados, foram identificados 9 artigos científicos e 7 papers/apresentações (conferências), 2 slides, 4 blogs e 2 capítulos de livro (Quadro 1). Na categoria "Tipo de estudo": 10 são artigos de pesquisa, 7 artigos de revisão, 1 estudo de caso, 2 livros e 4 apresentam opiniões de experts postadas em blogs ou sites de instituições.

\section{Quadro 1 - Research Data Science: documentos analisados}

\begin{tabular}{|c|c|c|}
\hline Id & Autoria & Título \\
\hline 1 & AIMS (2017) & $\begin{array}{l}\text { Responsible Data Science. Ensuring fairness, } \\
\text { accuracy, confidentially, transparency }\end{array}$ \\
\hline 2 & de Jong et al. (2018) & $\begin{array}{l}\text { CLARIN: towards FAIR and Responsible Data Science } \\
\text { using language resources }\end{array}$ \\
\hline 3 & de Smedt et al. (2018) & $\begin{array}{l}\text { Towards an open science infrastructure for the digital } \\
\text { humanities }\end{array}$ \\
\hline 4 & Fišer, Lenardic, e Erjavec (2018) & CLARIN's Key resource families \\
\hline 5 & GE 301 Group 7. (2017) & Responsible Data Science \\
\hline 6 & Kemper Kolkman (2018) & $\begin{array}{l}\text { Transparent to whom? No algorithmic accountability } \\
\text { without a critical audience }\end{array}$ \\
\hline 7 & Moerel (2016) & $\begin{array}{l}\text { GDPR conundrums - the data protection officer } \\
\text { requirement }\end{array}$ \\
\hline 8 & Ohm (2014) & $\begin{array}{l}\text { Changing the rules: general principles for data use and } \\
\text { analysis }\end{array}$ \\
\hline 9 & Piersma (s.d.) & Data in urban environments \\
\hline 10 & $\begin{array}{l}\text { Srivastava, Scannapieco, e Redman } \\
\text { (2019) }\end{array}$ & $\begin{array}{l}\text { Ensuring high-quality private data for Responsible } \\
\text { Data Science }\end{array}$ \\
\hline 11 & Stoyanovich et al. (2017) & $\begin{array}{l}\text { Fides: towards a platform for Responsible Data } \\
\text { Science }\end{array}$ \\
\hline 12 & Stoyanovich et al. (2018) & Panel: a debate on data and algorithmic ethics \\
\hline 13 & Stoyanovich e Howe (2018) & Follow the Data! \\
\hline
\end{tabular}


Morgana Carneiro Andrade, Paula Regina Gonçalez, Decio Wey Berti Junior, Ana Alice Baptista, Caio Saraiva Coneglian

Ciência responsável dos dados: imparcialidade, precisão, confidencialidade, e transparência dos dados

\begin{tabular}{|l|l|l|}
\hline Id & Autoria & Título \\
\hline 14 & $\begin{array}{l}\text { Stoyanovich, Howe, e Jagadish } \\
(2018)\end{array}$ & $\begin{array}{l}\text { Special session: a technical research agenda in data } \\
\text { ethics and responsible data management }\end{array}$ \\
\hline 15 & Stoyanovich, Yang, e Jagadish (2018) & On line set selection with fairness and diversity \\
\hline 16 & Taylor (2018) & Data, visibility and justice \\
\hline 17 & Taylor e Broeders (2015) & In the name of development \\
\hline 18 & van Berchum e Trippel (2015) & CLARIN data management Activities \\
\hline 19 & van der Aalst (2016) & Green Data Science \\
\hline 20 & van der Aalst et al. (2018) & $\begin{array}{l}\text { Views on the past, present, and future of business } \\
\text { information systems engineering }\end{array}$ \\
\hline 21 & van der Aalst et al. & $\begin{array}{l}\text { Responsible data science: using event data in a } \\
\text { "people friendly manner" }\end{array}$ \\
\hline 22 & van der Hoven (2018) & SoBigData ethics unpacking privacy \\
\hline 23 & Veuger (2017) & Attention to disruption and blockchain creates \\
\hline 24 & Veuger (2018) & $\begin{array}{l}\text { Trust in a viable real estate economy with disruption } \\
\text { and blockchain }\end{array}$ \\
\hline
\end{tabular}

Fonte: Autores

Apesar de os relatos de experiências ainda serem poucos, orientações e ações que podem atender às diretrizes FACT são apresentadas pela maior parte das publicações consultadas. A seguir, expomos direcionamentos e iniciativas que podem contribuir para a aplicação do FACT (Quadros 2 e 3).

\section{Quadro 2 -Diretrizes e Iniciativas FACT}

\section{FAIRNESS}

O repositório Clarin inclui ações como contrato de licença de usuário final, termos de serviços dos dados, adição de restrições e responsabilidades para o usuário final, principalmente no que se refere à privacidade (3)

O repositório Clarin requer a proveniência dos dados a partir dos metadados, que possibilitam a rastreabilidade dos dados e a inclusão de PIDs que viabilizam a citação a e replicabilidade dos dados. Está em desenvolvimento a inclusão de amostras melhoradas, enriquecimento dos dados com o uso de metadados (extralinguísticos), ligação com fontes externas (ex. Gazetters) e anotações em nível conceitual (3)

No domínio das humanidades digitais, a utilização de dados deverá ser realizada com a preocupação de evitar dados tendenciosos que inflinjam a privacidade, a confidencialidade e a transparência de modo a comprometer os resultados por ausência de confiabilidade (3)

Se os dados de treinamento são tendenciosos ou têm erros, é lógico que o resultado algorítmico seja injusto ou errado (14)

Três pilares são apontados como base para a imparcialidade internacional de dados: (in)visibilidade, (des)engajamento com tecnologia e antidiscriminação Esses pilares integram direitos positivos e negativos e liberdades (16)

A economia política de dados prevê o envolvimento metodológico em que se inclui a identificação sobre o que é, quem é importante e como eles se relacionam com os resultados desejados (16)

As técnicas de ciência de dados precisam garantir justiça. As decisões e percepções automatizadas não devem ser usadas para discriminar de maneira que são inaceitáveis do ponto de vista legal ou ético (19)

A prevenção da discriminação com a criação de algoritmos de decisão automatizados que não discriminam, usando variáveis sensíveis (sexo, idade, nacionalidade etc.), utilizando um método predefinido; pré-processamento ou pós-processamento (19)

\section{ACCURACY}

Os líderes devem defender as abordagens a priori de gestão de dados (10) 
Morgana Carneiro Andrade, Paula Regina Gonçalez, Decio Wey Berti Junior, Ana Alice Baptista, Caio Saraiva Coneglian

Ciência responsável dos dados: imparcialidade, precisão, confidencialidade, e transparência dos dados

O compartilhamento de dados com preservação de privacidade utiliza Secure Multiparty Computation (SMC) (10)

Os cientistas de dados devem enfatizar a abordagem qualitativa adotando métodos e técnicas a priori para avaliar a qualidade de dados (10)

Os algoritmos para várias configurações de problemas com dados de streaming são desenvolvidos e uma decisão on-line deve ser feita em cada item à medida que forem apresentados (15)

O uso de Big Data envolve a análise remota para tornar populações visíveis. Tal visibilidade cria uma força sobre os assuntos dos dados por meio de volume de dados ao invés de precisão (accuracy) e detalhe (17)

A incerteza dos dados em Big Data está relacionada com o volume, velocidade, variedade e veracidade. A veracidade está associada à confiabilidade dos dados de entrada. Para melhor interpretação dos resultados das análises, podem ser adotados a imprecisão explícita ou os diagnósticos de confiança mais explícitos (19)

\section{CONFIDENTIALITY}

O uso de dados tendenciosos viola a privacidade ou confiabilidade, e a ausência de transparência pode distorcer conclusões e afetar as relações de confiança (2)

As licenças podem ser estabelecidas entre provedor de dados, repositórios e usuários finais em que se incluem restrições e responsabilidades e assegurem o compromisso da utilização de dados bem descritos, como forma de resguardar a privacidade (2)

A criação de leis de privacidade deveria abranger o contexto de determinado conteúdo e escopo adequado (8)

O controle de acesso à identidade e/ou papel do indivíduo que busca a identificação e o acesso aos dados precisa se efetivar por meio de uma política e de tecnologia (10)

$\mathrm{O}$ anonimato de dados, a partir do uso de modelo de privacidade diferencial local (LDP), combina a garantia estatística de privacidade diferencial e a segurança de que a informação nunca será visível em sua forma bruta (10)

Os empresários devem focar o potencial dos mercados de dados que equilibram a variação dos preços com os trade-offs de qualidade de dados privados (12)

Os conjuntos de dados sintéticos que preservam a privacidade, quando apropriados, podem ser liberados em vez de conjuntos de dados reais para expor certas características dos dados (ex.: conjuntos de dados reais sensíveis) (13)

Quando apropriados, conjuntos de dados sintéticos que preservam a privacidade podem ser liberados em vez de conjuntos de dados reais para expor certas características dos dados, se os conjuntos de dados reais forem sensíveis e não puderem ser liberados para o público (13)

A análise e visualização de dados são realizadas pela abordagem de banco de dados gráficos (Graph Vision) e implementação de machine learning como tecnologias de análise de dados (5)

O sistema de avaliação de Impacto Privado (PIA) ajuda a entender melhor como as informações pessoais podem ser usadas, armazenadas, compartilhadas e também diminui os riscos de privacidade (5)

A confidencialidade dos dados é um dos aspectos mais difíceis de se atingir. Os princípios de proteção de dados não devem ser aplicados a informações anônimas ou que não estejam relacionadas com uma pessoa física identificada ou identificável ou com dados tornados anônimos de forma que não seja mais possível identificar a pessoa (19)

A confidencialidade pode estar ameaçada ao longo do processo de análise dos resultados. É sugerido o uso de métodos de desidentificação como: remoção de variáveis, randomização, hashing, shuffling, subamostragem, agregação, truncamento, generalização, adição de ruído, entre outros. Não obstante, essas ações podem impactar a qualidade dos resultados (19)

$O$ artigo versa sobre o General Data Protection Regulation (GDPR) e os requisitos para a contratação de um Data Protection Officer (DPO) propõem uma coleção de técnicas e abordagens que buscam facilitar o acesso aos benefícios da Ciência de Dados e Big Data enquanto garante imparcialidade, confidencialidade, precisão (correção) e transparência (21)

As diretrizes para a privacidade são: consentimento informado, direito de ser esquecido, gerenciamento de identidade, privacidade recíproca, granulação grosseira, anonimização, vigilância, contravigilância, detecção de violação / intrusão, aplicativos de Big Data para detectar violações de privacidade em Big Data (21)

\section{TRANSPARENCY}

O registro de dados de proveniência nos metadados CLARIN torna os dados rastreáveis e o uso de PIDs torna os dados passíveis de serem citados e seu uso replicável (3) 
Morgana Carneiro Andrade, Paula Regina Gonçalez, Decio Wey Berti Junior, Ana Alice Baptista, Caio Saraiva Coneglian

Ciência responsável dos dados: imparcialidade, precisão, confidencialidade, e transparência dos dados

As etapas de conversão de dados devem ser explicitadas para o resultado final na forma de pipeline (5)

Uma infraestrutura de pesquisa pode ser considerada "responsável" apenas se acomoda usuários de dados, controladores e processadores como responsáveis pela produção de resultados epistêmicos (6)

As agências governamentais devem disponibilizar publicamente resumos com as propriedades estatísticas relevantes do conjunto de dados que possam auxiliar na tomada de decisão mediante a preservação de dados e a privacidade dos indivíduos

Os técnicos devem construir a priori métodos de qualidade de dados na Internet das Coisas; ferramentas para limpeza, transporte e integração de dados (10)

As abordagens qualitativas podem ajudar a elaborar relatos detalhados e ricos do uso de modelos algorítmico, possibilitando a análise do contexto em que eles se inserem (10)

As diretrizes FACT devem ser aplicadas desde a fase em que os cientistas recebem os dados, passando pela fase de disputa de dados, modelagem, implantação até a interpretação dos resultados do modelo algorítmico (11)

O cientista de dados deve trabalhar de forma responsável nas fases de recebimento, manipulação, modelagem e implantação de dados e deve persistir quando os resultados do modelo algorítmico forem interpretados e o modelo algorítmico mantido (11)

As agências devem disponibilizar ao público informações sobre a coleta de dados e a metodologia de pré-processamento, em termos de suposições, critérios de inclusão, fontes de viés conhecidas e qualidade dos dados (13)

Os dados coletados devem ser mantidos e disponibilizados para tornar o processo de tomada de decisão transparente (13)

A interpretabilidade deve apresentar as propriedades estatísticas de um conjunto de dados e a metodologia usada para produzi-los. Em última análise, fundamentar sua "adequação ao uso" no contexto de um sistema ou tarefa de decisão automatizada específica é fundamental para a transparência dos dados (13)

As agências devem: a) disponibilizar ao público informações sobre a coleta de dados e a metodologia de pré-processamento, em termos de suposições, critérios de inclusão, fontes de viés conhecidas e qualidade dos dados; b) anotar adequadamente os conjuntos de dados, quando eles forem compartilhados, e manter informações sobre como os conjuntos de dados são adquiridos e manipulados; c) explicar as propriedades estatísticas dos conjuntos de dados; d) descobrir fontes de preconceitos e fazer declarações sobre qualidade de dados e adequação para uso (13)

A aprovação de transparência algorítmica é apontada como uma forma de proporcionar a transparência (13)

As agências devem disponibilizar resumos publicamente disponíveis de propriedades estatísticas relevantes dos conjuntos de dados que possam auxiliar na interpretação das decisões tomadas com os dados, aplicando métodos de última geração para preservar os dados e a privacidade dos indivíduos, além de liberar conjuntos de dados de treinamento e validação sempre que possível (13)

A transparência do algoritmo não é suficiente para garantir a precisão dos resultados. É preciso conhecer os dados utilizados (14)

A transparência dos dados, por si só, não garante o sucesso das tomadas de decisões. São necessárias a contextualização e a literacia de dados, por parte dos gestores, que impactem negativamente nas decisões de gestores e legisladores (14)

As decisões (baseadas em model os de processo ou padrões frequentes e regras de decisão automatizadas) e os resultados da análise devem ser transparentes para garantir a aceitação e o uso adequado das técnicas de ciências dos dados (19)

A transparência deve envolver a tomada de decisão automatizada, a explicação de decisões individuais, a inteligibilidade, clareza e a compreensão dos resultados da análise. O acesso aos dados e a técnica de análise utilizada devem ser explicitados por meio de link (19)

A existência de um vínculo claro entre os dados e os resultados (histórias da análise) favorece a transparência. É necessário o detalhamento e a inspeção dos dados na perspectiva do modelo, de modo que seja possível reproduzir os resultados da análise dos dados originais (19)

A geração de linguagem natural é utilizada para transformar os resultados da análise selecionada em relatórios individualizados, concisos e fáceis de ler (19)

Os cientistas sociais devem contribuir para o entendimento das relações de confiança e qualidade de dados e para a pertinência da Ciência Responsável de Dados (21)

Inf. Inf., Londrina, v. 25, n. 2, p. 26 - 48, abr./jun. 2020. 


\section{Nota: *() Identificação da fonte relacionada no Quadro 1.}

Fonte: Autores

Ainda que de importância ímpar para que sejam atendidas questões referentes à ética, à economia e aos aspectos sociais, é tímida a sinalização das diretrizes do FACT na literatura. Nos artigos consultados, a transparência é o princípio mais comentado pela maior parte dos artigos consultados, o que denota a preocupação dos autores com a demanda da sociedade sobre o tema, intimamente relacionado com a disponibilização pública de dados. O princípio da confidencialidade foi o segundo mais abordado, seguido por imparcialidade e precisão. Ao analisar esses resultados, observamos que existem dificuldades em adotar os princípios de forma igualitária e eficaz. Como exemplo, têm-se as ações que, ao preservarem a confidencialidade e a imparcialidade, incidem nos níveis de transparência.

O posicionamento de Kemper e Kolkman (2018) é que deve haver mais estudos empíricos sobre modelos algorítmicos para testar a responsabilidade algorítmica, pois só assim será possível desenvolver diretrizes sólidas adequadas às práticas existentes. Esse deve ser o grande desafio, já que a existência de legislação não assegura a efetividade do uso desses princípios (Moerel, 2016, Ohm, 2014).

De acordo com Ohm (2014), a maioria das leis de privacidade se concentra na coleta e na divulgação de dados, e não no uso. Por outro lado, ainda existem diferenças nas leis de um país para outro. A lei de privacidade dos Estados Unidos é diferente da Diretiva de Proteção de Dados da União Europeia, mesmo se tratando de aspectos cujos danos possam ser globais. A regra de que "[...] os dados devem ser processados para um fim específico e subsequetemente, usados ou comunicados apenas na medida que não são incompatíveis com a utilidade da transferência" também parece intangível. Trabalhar com grandes dados é trabalhar com o imprevisível. Sendo assim, como assegurar o resultado final da análise dos dados? Questionamento que motiva discussão e investigação no universo dos dados.

Por outro lado, agentes, como os analistas, programadores, cientistas de 
dados e o governo, têm papel imprescindível para que esses princípios sejam resguardados de forma a promover uma sociedade igualitária. No Quadro 3 são apresentadas algumas iniciativas relativas às diretrizes FACT. Como exposto neste estudo, as ações para garantir os quatro princípios são concebidas mais lentamente do que as tecnologias desenvolvidas para a análise e disseminação dos dados.

Pode-se observar que, em relação aos direcionamentos e iniciativas identificados na pesquisa, eles ainda demandam um grande esforço conjunto e interdisciplinar das áreas sociais e tecnológicas para que possam acontecer de fato. O lado social das nossas vidas é referido por Bauman (2001) como modernidade líquida, metáfora que ele adota para afirmar que tudo é fluído. Ou seja, Bauman considera que a modernidade que vivemos se caracteriza pela incapacidade de manter a forma.

Observando o período histórico que antecede essa fase, denominado por Bauman (2001) de "modernidade sólida", o autor apresenta ideias que apontam uma constância e segurança, pois a transformação de valores ocorria de forma lenta e perceptível, dando a sensação de certeza e controle sobre o que acontecia no mundo.

Assim, quando consideramos, por meio dessa abordagem da modernidade líquida, os direcionamentos que denotam mais o lado social, constatamos que eles podem ser mais complexos, especialmente em questões de discriminação e sensibilidade dos dados. De acordo com as iniciativas que mostram como vem sendo trabalhada a tecnologia em relação ao RDS, inferese que os sistemas de tratamento dos dados demandam maior flexibilidade no sentido de adaptar-se às vertiginosas mudanças nesse direcionamento, provocadas pelo fluxo resultante da pressão exercida pelas condições comuns à "vida líquida" (Bauman, 2001).

\section{CONCLUSÃO}

O conceito de RDS apresenta um significativo avanço no contexto do Big Data e da Data Science, trazendo importantes elementos que favorecem uma 
reflexão por parte dos cientistas de dados. Em especial, o FACT identifica claramente os aspectos e facetas vinculados a esse conceito, bem como demonstra o que os cientistas de dados devem considerar durante os processos que compõem as fases do Ciclo de Vida dos Dados.

Observamos que as ações promovidas pelo grupo RDS, em relação à Green Data Science ou às diretrizes FACT, podem contribuir para salvaguardar os direitos individuais, sem que seja necessário adotar medidas que impeçam sumariamente $o$ acesso e a reutilização de dados.

Dessa forma, destacamos que as técnicas de análise de dados, em conjunto com as tecnologias de Internet das Coisas, vêm sendo amplamente utilizadas sob uma perspectiva tecnicista, visando a gerar valor para as organizações. Nesse sentido, o estudo e a aproximação das ciências sociais a essas temáticas são relevantes para que questões sociais sejam consideradas durante os processos tecnológicos. Embora haja importantes movimentos para aprovação de leis que garantam direitos individuais no contexto do Big Data, o uso da tecnologia é imprescindível para que esses direitos sejam assegurados.

Em vista do exposto, há necessidade de estudos empíricos sobre modelos de algoritmos, em que as medidas de transparência e imparcialidade sejam analisadas na prática, de forma que os envolvidos na geração, armazenamento e tratamento dos dados adotem mecanismos que lhes assegurem confiança quanto aos serviços prestados, assim como aos dados acessados.

O desenvolvimento de tal tipo de tecnologia de análise de dados, de acordo com o modelo FACT de abordagem de ciência responsável de dados, é de importância crucial, porque a falta de implementação dessa abordagem em tecnologias de dados pode resultar em consequências indesejáveis para a sociedade, causando impacto em três elementos: privacidade, segurança e responsabilidade.

Nota-se que dificilmente serão contemplados os quatro princípios FACT simultaneamente, entretanto deve-se buscar um equilíbrio em que sejam consideradas as compensações - priorizar um em detrimento de outro(s). No 
caso de garantir a confidencialidade, provavelmente, a precisão e a transparência não serão comprometidas. Logo, a busca por um equilíbrio entre esses quatro princípios deve ser almejada tanto pelos desenvolvedores como pelos gestores de dados.

\section{REFERÊNCIAS}

AIMS. Responsible data science. Ensuring, fairness, accuracy, confidentially, transparency. (2017). Disponível em:

http://aims.fao.org/activity/blog/responsible-data-science-ensuring-fairnessaccuracy-confidentially-transparency-fact. Acesso em: 10 Jan 2019.

ANNANY, M.; CRAWFORD, K. Seeing without knowing: limitations of the transparency ideal and its application to algorithmic accountability. New Media \& Soc., v. 20, n. 3, p. 973-989, 2016.

ÁVILA, F. B. S.J. Pequena enciclopédia de moral e civismo. Rio de Janeiro: MEC, 1967.

BAUMAN, Z. Modernidade líquida. Rio de Janeiro: Zahar, 2001.

COLLINS dictionary. (2019). Disponível em:

https://www.collinsdictionary.com/pt/dictionary/ english/accuracy. Acesso em: 2 mar. 2019.

DATA SCIENCE CENTER EINDHOVEN. Responsible Data Science. Ensuring fairness, accuracy, confidentiality \& transparency by design. (2019). Disponível em: https://www.tue.nl/en/research/research-areas/data-science/responsibledata-science/. Acesso em: Acesso em: 10 jan. 2019

DE JONG, F. M. G.; MAEGAARD, B.; DE SMEDT, K.; FIŠER, D.; VAN UYTVANCK, D. CLARIN: towards FAIR and responsible data science using language resources. In: Proceedings of the Eleventh International Conference on Language Resources and Evaluation (LREC 2018), 2018. p. 3259-3264. Disponível em: https://dspace.library.uu.nl/handle/1874/364776. Acesso em: 10 jan. 2019.

DE SMEDT, K.; DE JONG, F.; MAEGAARD, B.; FIŠER, D.; VAN UYTVANCK, $D$. Towards an open science infrastructure for the digital humanities: the case of CLARIN. CEUR-WS.org, v. 2084, p. 1-12, 2018. Disponível em: http://ceurws.org/Vol-2084/paper11.pdf. Acesso em: 10 jan. 2019.

EURONEWS. France fines Google $€ 50$ million using EU's transparency and consent law. R (2019). Disponível em: 
https://www.euronews.com/2019/01/21/france-fines-google-50-million-using-eus-transparency-and-consent-law. Acesso em: 10 Jan. 2019

EUROPEAN DATA SCIENCE ACADEMY. About EDSA. (2019). Disponível em: http://edsa-project.eu/overview/about-edsa/. Acesso em: 20 Jan. 2019.

FACEBOOK: Cambridge analytica data scandal. Wikipedia: The Free Encyclopedia. Apr. 26, 2019. Disponível em: https://en.wikipedia.org/wiki/Facebook\% E2\%80\%93Cambridge_Analytica_ data_scandal. Acesso em: 26 abr. 2019.

FIŠER, D.; LENARDIČ, J.; ERJAVEC, T. CLARIN's key resource families. In: Proceedings of the Eleventh International Conference on Language Resources and Evaluation (LREC-2018). 2018. Disponível em: http://www.lrecconf.org/proceedings//rec2018/pdf/829.pdf. Acesso em: 10 Jan. 2019.

GE 301 Group 7. Responsible Data Science. 2017. Disponível em: http://ge301.bilkent.edu.tr/fall2017group7/. Acesso em: 11 Jan. 2019.

GOLDIM, J. R. Confidencialidade (1997-2003). Disponível em: https://www.ufrgs.br/bioetica/confiden.htm. Acesso em: 15 Mar. 2019.

HAGGERTY, K.D.; ERICSON, R.V. The surveillant assemblage. Br. J. Sociol. v. 51, n. 4 , p. 605-622, 2000.

HILBERT, M.; LÓPEZ, P. The world's technological capacity to store, communicate, and compute information. Sciencexpress, p. 1-7, 2011. Disponível em:

http://www.ris.org/uploadi/editor/13049382751297697294Science-2011-Hilbertscience.1200970.pdf. Acesso em; 18 mar. 2019.

KEMPER, J.; KOLKMAN, D. Transparent to whom? No algorithmic accountability without a critical audience. Inf. Commun. Soc., p. 1-16, 2018. Disponível em: https://doi.org/10.1080/1369118X.2018.1477967. Acesso em. 23 jan. 2019.

LEPRI, B.; OLIVER, N.; LETOUZÉ, E.; PENTLAND, A.; VINCK, P. Fair, transparent, and accountable algorithmic decision-making processes the premise, the proposed solutions, and the open challenges. Philosophy and Technology. v. 31, n. 4, p. 611-627, 2018. doi: 10.1007/s13347-017-0279-x.

LODDER, G. M. A.; SCHOLTER, R. H. J.; GOOSSENS, L.; ENGELS, R. C. M. E.; VERHAGEN, M. Loneliness and the social monitoring system: emotion recognition and eye gaze in a real-life conversation. Br. J. Psychol., v. 107, n. 1, p. 135-153, 2016. doi: https://doi.org/10.1111/bjop.12131 
MOEREL, L. GDPR conundrums: the data protection officer requirement. 19 July 2016. Disponível em: https://research.tilburguniversity.edu/en/ publications/gdpr-conundrums-the-data-protection-officer-requirement. Acesso em: 23 jan. 2019.

MOEREL, L.; PRINS, C. Privacy for the homo digitalis: proposal for a new regulatory framework for data protection in the light of Big Data and the Internet of Things. May 25, 2016. Acesso em: 10 jan. 2019. doi: http:??dx.doi.org/10.2139/ssrn.2784123.

OHM, P. Changing the rules: general principles for data use and analysis. In: LANE, J.; STODDEN, V.; BENDER, S.; NISSENBAUM, H. (Ed.). Privacy, big data, and the public good: frameworks for engagement. Cambridge: Cambridge University Press, 2014. v.1, p. 96-111.

PENNOCK, M. Digital curation: a life-cycle approach to managing and preserving usable digital information. Library \& Archives Journal, n. 1. 2007. Disponível em:

http://www.ukoln.ac.uk/ukoln/staff/m.pennock/publications/docs/libarch_curation.pdf. Acesso em: 12 May 2019.

PIERSMA, N. Data in urban environments. In: PIERSMA, N. (Ed.). Through the clouds: urban analytics for smart cities. Amsterdam: Hogeschool van Amsterdam, 2018. p. 11-21.

SATARIANO, A. Google is fined $\$ 57$ million under Europe's Data Privacy Law. New York Times. Disponível em:

https://www.nytimes.com/2019/01/21/technology/google-europe-gdpr-fine.html. Acesso em: 21 mar. 2019.

SRIVASTAVA, D.; SCANNAPIECO, M.; REDMAN, T. C. Ensuring high-quality private data for responsible data science: vision and challenges. ACM Journal of Data and Information Quality (JDIQ). v. 11, n. 1, p. 1, 2019.

STOYANOVICH, J.; HOWE, B. Follow the data! Algorithmic transparency starts with data transparency. 2018. Disponível em:

https://ai.shorensteincenter.org/ideas/2018/11/26/follow-the-data-algorithmictransparency-starts-with-data-transparency. 21 mar. 2019.

STOYANOVICH, J.; HOWE, B.; JAGADISH, H. V. Special Session: A technical research agenda in data ethics and responsible data management. In Proceedings of the 2018 International Conference on Management of Data, p. 1635-1636, 2018.

STOYANOVICH, J.; HOWE, B.; ABITEBOUL, S.; MIKLAU, G.; SAHUGUET, A.; WEIKUM, G. Fides: Towards a platform for responsible data science. In: SSDBM'17. 29th International Conference on Scientific and Statistical Database Management, Jun 2017, Chicago, United States. 
10.1145/3085504.3085530. hal-01522418. Disponível em:

https://hal.inria.fr/hal-01522418/document. Acesso em: 10 jan. 2019.

STOYANOVICH, J.; HOWE, B.; JAGADISH, H. V.; MIKLAU, G. Panel: a debate on data and algorithmic ethics. Proceedings of the VLDB Endowment, v. 11, $\mathrm{n}$. 12, p. 2165-2167, 2018.

STOYANOVICH, J.; YANG, K.; JAGADISH, H. V. Online set selection with fairness and diversity constraints. In Proceedings of the 21st International Conference on Extending Database Technology (EDBT), March 26-29, 2018. Disponível em: https://openproceedings.org/2018/conf/edbt/paper-98.pdf. Acesso em: 10 jan. 2019.

TAYLOR, L. What is data justice? The case for connecting digital rights and freedoms globally. Big Data \& Society. p. 1-14, July/Dec. 2017 Disponível em: https://doi.org/10.1177/2053951717736335. Acesso em: 10 jan. 2019.

TAYLOR, L. Data, visibility and justice. 2017. Disponível em: https://redasci.org/wp-content/uploads/2016/10/Linnet-Taylor-RDS-16.3.17.pdf. Acesso em: 10 jan. 2019.

TAYLOR, L.; BROEDERS, D. (2015). In the name of development: power, profit and the datafication of the global south. Geoforum, v. 64, p. 229-237, 2015.

VAN BE.RCHUM, M.; TRIPPEL, T. CLARIN data management activities in the PARTHENOS context. In CLARIN Annual Conference 2018. pp. 95-99, 2018. Disponível em: https://ris.utwente.nl/ws/portalfiles/portal/63914609/CE_2018_1292_CLARIN20 18_ConferenceProceedings.pdf\#page=102. Acesso em: 10 jan. 2019.

VAN DER AALST, W. M. Green data science: using big data in an" environmentally friendly" manner. In 18th International Conference on Enterprise Information Systems (ICEIS 2016). Apr. 25-28, 2016. Rome: SciTePress, 2016. p. 9-21. Disponível em: https://pdfs.semanticscholar.org/5889/68dd392ae93b1524aa7a491917d839bca 050.pdf. Acesso em: 10 jan. 2019.

VAN DER AALST, W. M.; BECKER, J.; BICHLER, M.; BUHL, H. U.; DIBBERN, J.; FRANK, U., ... HUI, K.-L. et al. Views on the past, present, and future of business and information systems engineering. Bus. Inf. Syst. Eng., v. 60, n. 6, p. 448-450, 2018. Disponível em: https://doi.org/10.1007/s12599-018-0561-1. Acesso em: 10 jan. 2019.

VAN DER AALST, W. M.; BICHLER, M.; HEINZL, A. Responsible data science. Bus. Inf. Syst. Eng., v. 59, n. 5, p. 311-313, 2017. Disponível em: https://link.springer.com/article/10.1007\%2Fs12599-017-0487-z. Acesso em: 10 jan. 2019. 
VAN DER HOVEN, J. SoBigData ethics unpacking privacy designing for responsibility. Disponível em: https://slideplayer.com/slide/13113648/. Acesso em: 10 jan. 2019.

VEUGER, J. Attention to disruption and blockchain creates a viable real estate economy. J. US-China Public Adm., v. 14, n. 5, p. 263-285, 2017. Disponível em: https://davidpublisher.org/Public/uploads/Contribute/ 5a3c644925d78.pdf. Acesso em: 10 jan. 2019.

VEUGER, J. (2018). Trust in a viable real estate economy with disruption and blockchain. Facilities, v. 36, n. 1/2, p. 103-120, 2018. Disponível em: https://doi.org/10.1108/F-11-2017-0106. Acesso em: 10 jan. 2019.

WILKINSON, M. D.; DUMONTIER, M.; MONS, B. The FAIR guiding principles for scientific data management and stewardship. Scientific Data, v. 3, n. 160018, 2016. Acesso em: 10 jan. 2019.

\title{
RESPONSIBLE DATA SCIENCE: IMPARTIALITY, ACCURACY, CONFIDENTIALITY AND TRANSPARENCY OF DATA
}

\begin{abstract}
Introduction: In the Big Data context, as an urgent need arises the application of individual and corporate rights and regulatory standards that safeguard privacy, impartiality, accuracy and transparency. In this scenario, Responsible Data Science emerges as an initiative based on the FACT guidelines, which correspond to the adoption of four principles: impartiality, accuracy, confidentiality and transparency. Objective: To address alternatives that can ensure the application of the FACT guidelines. Methodology: An exploratory and descriptive research with a qualitative approach was developed. Searches were performed on the Web of Science, Scopus, and Scholar Google search engines using Responsible Data Science, Fairness, Accuracy, Confidentiality, Transparency Data Science, FACT, and FAT related to Data Science. Results: Responsible Data Science emerges as an initiative based on the FACT guidelines, which correspond to the adoption of the principles: impartiality, accuracy, confidentiality and transparency. In implementing these guidelines, consideration should be given to the use of techniques and approaches being developed by Green Data Science. Conclusions: It is concluded that Green Data Science and the FACT guidelines contribute significantly to safeguarding individual rights and that no measures need to be taken to prevent access and reuse of data. Challenges for implementing the FACT guidelines require studies, sine qua non conditions for tools for data analysis and dissemination to be developed at the design stage of methodologies.
\end{abstract}

Descriptors: Data science. Ethic. Big data. Responsible Data Science. 


\title{
GARANTIZAR LA IMPARCIALIDAD, LA EXACTITUD, LA CONFIDENCIALIDAD Y LA TRANSPARENCIA DE LOS DATOS EN LA PERSPECTIVA DE LA CIENCIA DE LOS DATOS
}

\begin{abstract}
RESUMEN
Introducción: en el contexto de Big Data, como una necesidad urgente surge la aplicación de los derechos individuales y corporativos y las normas reguladoras que salvaguardan la privacidad, imparcialidad, precisión y transparencia. En este escenario, Responsible Data Science surge como una iniciativa basada en las pautas de FACT, que corresponden a la adopción de cuatro principios: imparcialidad, precisión, confidencialidad y transparencia. Objetivo: abordar alternativas que puedan garantizar la aplicación de las pautas de FACT. Metodología: se desarrolló una investigación exploratoria y descriptiva con un enfoque cualitativo. Las búsquedas se realizaron en los motores de búsqueda de Web of Science, Scopus y Scholar Google utilizando los términos "Ciencia de datos responsable", "Justicia, precisión, confidencialidad, transparencia + ciencia de datos", FACT y FAT relacionados con ciência de los datos. Resultados: Responsible Data Science surge como una iniciativa basada en los lineamientos de FACT, que corresponden a la adopción de los principios: imparcialidad, precisión, confidencialidad y transparencia. Al implementar estas pautas, se debe considerar el uso de técnicas y enfoques desarrollados por Green Data Science. Conclusiones: Se concluye que Green Data Science y las pautas FACT contribuyen significativamente a salvaguardar los derechos individuales y que no es necesario tomar medidas para evitar el acceso y la reutilización de datos. Los desafíos para implementar las pautas FACT requieren estudios, condiciones sine qua non para desarrollar herramientas para el análisis y la difusión de datos en la etapa de diseño de las metodologias
\end{abstract}

Descriptors: Ciencia de datos. Ética. Big data. Ciencia responsable de datos.

Recebido em: 15.11 .2019

Aceito em: 20.04.2020 